\title{
Framework for Energy Efficiency Analysis of Wireless Networks
}

\author{
(Invited Paper) \\ Gunther Auer*, Vito Giannini ${ }^{\dagger}$, Magnus Olsson ${ }^{\S}$, Manuel J. Gonzalez ${ }^{* *}$, Claude Desset ${ }^{\dagger}$ \\ *DOCOMO Euro-Labs, 80687 Munich, Germany, Email: auer@docomolab-euro.com \\ ${ }^{\dagger}$ IMEC, Leuven, Belgium \\ $\S$ Ericsson Research, SE-164 80 Stockholm, Sweden \\ ** TTI (Technologies of Telecommunication and Information), 39011 Santander, Spain
}

\begin{abstract}
In order to quantify the energy savings in wireless networks, the power consumption of the entire system needs to be captured and an appropriate energy efficiency evaluation framework must be defined. In this paper, the necessary enhancements over existing performance evaluation frameworks are discussed, such that the energy efficiency of the entire network comprising component, node and network level contributions can be quantified. This includes a sophisticated power model for various base station (BS) types, which maps the RF output power radiated at the antenna elements to the total supply power of a BS site. The proposed evaluation framework is applied to quantify the energy efficiency of the downlink of a 3GPP LTE radio access network.
\end{abstract}

Index Terms-Energy efficiency, green radio, power model, system level energy efficiency evaluations, energy aware radio and network technologies (EARTH)

\section{INTRODUCTION}

The global mobile communication industry is growing rapidly. Today there are already more than 4 billion mobile phone subscribers worldwide [1], more than half the entire population of the planet. Obviously, this growth is accompanied by an increased energy consumption of mobile networks. Global warming and heightened concerns for the environment of the planet require a special focus on the energy efficiency of these systems [2-4]. The EARTH ${ }^{1}$ project $[3,5]$ is a concerted effort to achieve this goal and as part of its objectives, a holistic framework is developed to evaluate and compare the energy efficiency of several design approaches of wireless cellular communication networks.

The widely accepted state of the art to evaluate the performance of a wireless network is to simulate the relevant aspects of the radio access network (RAN) at system level. The computed results are, e.g. the system throughput measured in bit/s, quality of service (QoS) metrics, and fairness in terms of cell-edge user throughput. In order to ensure that the results generated by different RAN system simulation tools are comparable, well defined reference systems and scenarios are specified. This is an outcome of extensive consensus work from standardization bodies, such as 3 GPP $[6,7]$, and international research projects, such as the EU project Wireless

\footnotetext{
${ }^{1}$ EU funded research project EARTH (Energy Aware Radio and neTwork tecHnologies), FP7-ICT-2009-4-247733-EARTH, Jan. 2010 to June 2012. https://www.ict-earth.eu
}

World Initiative New Radio (WINNER) [8], with partners from academia as well as from industry. The most recent example is the global effort in ITU to evaluate system proposals for compliance with IMT-Advanced requirements [9]. In that direction, the EARTH $\mathrm{E}^{3} \mathrm{~F}$ builds on the 3GPP evaluation framework for LTE [6].

The EARTH $E^{3} F$ presented in Section 2 facilitates the assessment of the overall energy efficiency of cellular networks by incorporating a sophisticated power model, introduced in Sections 3, into existing system level simulation tools. Then, using the metrics defined in Section 4, in Section 5 the $\mathrm{E}^{3} \mathrm{~F}$ is applied in order to provide an exemplary assessment of the base station (BS) energy efficiency of 3GPP LTE.

\section{ENERgy EfFiciency Evaluation Framework $\left(E^{3} \mathrm{~F}\right)$}

The EARTH $\mathrm{E}^{3} \mathrm{~F}$ illustrated in Fig. 1, identifies the essential building blocks, such that the energy efficiency of the entire network, comprising component, node and network level can be quantified. An accurate holistic assessment of integrated solutions does essentially require a dynamic system level simulator, where time variations of the served traffic, the radio resource management (RRM) and the PHY are modeled on an OFDM symbol basis. Although the specific realization of a system level simulation tool largely depends on the specific problem at hand, as well as the chosen software implementation, it is envisaged that for the assessment of combinations of $e$

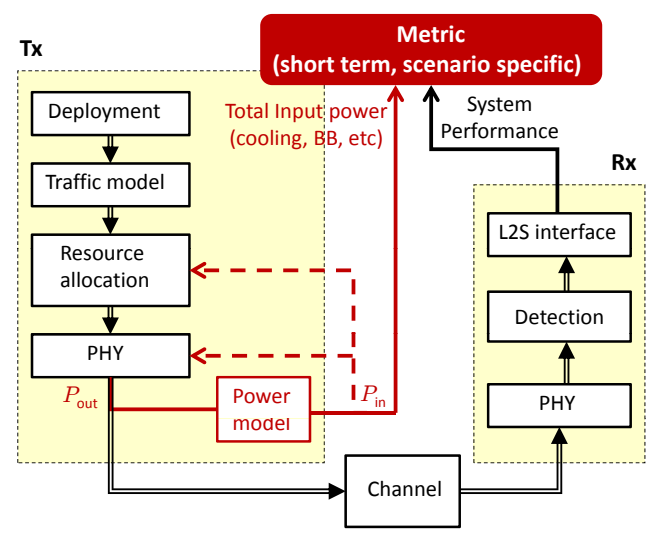

Fig. 1. EARTH Energy efficiency evaluation framework $\left(E^{3} F\right)$. 
unified system concept, the $\mathrm{E}^{3} \mathrm{~F}$ should capture the following aspects:

- Unlike conventional frameworks that are optimized for capacity and evaluated at full load, for energy efficiency evaluations the system performance is to be assessed at low load scenarios [5].

- A sophisticated power model (specified in Section 3), that maps the RF output power radiated at the antenna elements to the total supply power of a BS site. The power model maps the gains on the component level (e.g. an improvement of the energy efficiency of the power amplifiers) to energy savings on the entire network.

- Appropriate energy efficiency metrics as discussed in Section 4.

An $\mathrm{E}^{3} \mathrm{~F}$ compliant system level simulation tool must be able to capture approaches where the resource utilization and/or the radio transmission part (block PHY in Fig. 1) are operated in an energy aware way, i.e. resource allocation and modulation scheme are adapted with respect to the required BS input power, $P_{\text {in }}$, as opposed to the transmitted RF output power $P_{\text {out }}$ at the antenna elements (marked by dashed lines in Fig. 1).

The basic building blocks of a dynamic system level simulator, as shown in Fig. 1, are briefly described in the following.

Deployment: specifies the deployment scenario (dense urban, urban, suburban and rural), the BS specification (antenna configuration, maximum transmission power, etc.) and the BS site locations, e.g. hexagonal macro-cellular layout consisting of 19 sites, with 3 sectors per site. Mobile users are randomly dropped, following a uniform distribution, or clustered nonuniform distribution to resemble hot spots [6]. This block includes the association of mobile users to their serving BS, typically the BS with the highest average channel gain.

Traffic model: instantaneous traffic variations per mobile user, described by statistical traffic models, e.g. FTP file download or VoIP calls [6].

Resource allocation: the assignment of physical resource blocks (PRBs) to mobile users in time, frequency and space, to be generated for all BS sites in the network.

$P H Y$ : includes the framing/deframing operation at the transmitter/receiver, inserting control information and reference signals (pilots) to facilitate channel estimation. Furthermore, MIMO processing and modulation are performed. For system level evaluation purposes, the PHY is typically subject to abstractions, so as to avoid the complexity of detailed baseband processing (e.g. channel coding, channel estimation, etc.).

Power model: defines the interface between component and system level. The power model maps the consumed input power, $P_{\text {in }}$, to achieve a certain RF output power, $P_{\text {out }}$, at the antenna, as is elaborated in detail in Section 3.

Channel: comprises all transmitter-to-receiver links (for the downlink all BSs to all mobile links) in the network. This includes the intended links, as well as the interference between adjacent cells. The channel of one link is composed of distance dependent path-loss, log-normal shadowing and timevariant frequency-selective fading. For signals that penetrate into buildings, wall penetration losses are to be added [6]. In case of MIMO transmission, one channel realization is to be generated between each transmit and receive antenna element.

Detection: the signal to interference plus noise ratio (SINR) for each transmitted symbol is determined.

Link to system (L2S) interface: given the SINR per symbol per user, the desired figures of merit are determined, e.g. user throughput, block error rates (BLER), delay, etc. Different mappings between the input (SINR) and the output, and their respective accuracy, are discussed in [10].

\section{POWER MODEL}

The power model constitutes the interface between component and system level, which allows quantifying how energy savings on specific components enhance the energy efficiency at system level.

\section{A. Base Station Power Consumption Breakdown}

This section provides a power model for various types of LTE Base Stations. Fig. 2 shows a simplified block diagram of a complete BS that can be generalized to all BS types, including macro, micro, pico and femto BSs. In general, it is composed of multiple transceivers (TRXs) for each transmit/receive antenna element. Each of these TRXs comprises a lossy Antenna Interface (AI), a Power Amplifier (PA), a Radio Frequency (RF) small-signal transceiver section and a baseband (BB) interface, both including a receiver (uplink) and transmitter (downlink), a DC-DC power supply, an active cooling system and an AC-DC unit for connection to the electrical power grid. Note that active cooling is only relevant for macro BS, while it is omitted in smaller BS.

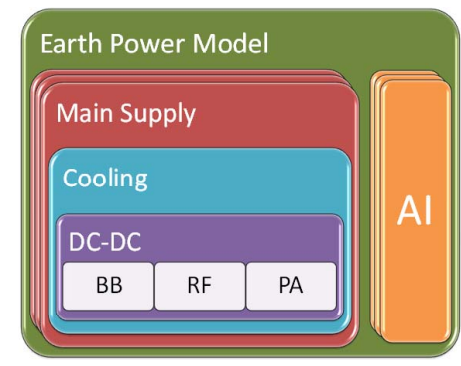

Fig. 2. Block diagram of a base station transceiver.

In the following subsections, the various TRX parts are analyzed.

Antenna Interface (AI): The influence of the antenna type on power efficiency is modeled by a certain amount of losses, including the feeder (where relevant), antenna bandpass filters, duplexers, and matching components.

Power Amplifier (PA): Typically, the most efficient operating point of a power amplifier is close to the maximum output power (near saturation). Unfortunately, non-linear effects and OFDM modulation with non-constant envelope signals force the power amplifier to operate in a more linear region, i.e., 6 to $12 \mathrm{~dB}$ below saturation [11]. This prevents Adjacent Channel Interference (ACI) due to non-linear distortions, and therefore avoids performance degradation at the receiver. However, this 
high operating back-off gives rise to poor power efficiency. Digital techniques such as clipping and digital-pre-distortion in combination with Doherty PAs improve the power efficiency, while keeping ACI under control, but require an extra feedback for pre-distortion and significant additional signal processing. While these techniques are necessary in macro and micro BSs, they are not used in smaller BSs, as the PA power consumption accounts for a smaller percentage of the power breakdown, allowing for a higher operating back-off.

The Small-Signal RF Transceiver (RF TRX) comprises a receiver and a transmitter for uplink (UL) and downlink (DL) communication. The linearity and blocking requirements of the RF TRX may differ significantly depending on the BS type, and so its architecture. Typically, low-IF (IntermediateFrequency) or super-heterodyne architectures are the preferred choice for macro/micro BSs, whereas a simpler zeroIF architecture are sufficient for pico/femto BSs. Parameters with highest impact on the RF TRX energy consumption are the required bandwidth, the allowable Signal-to-Noise And Distortion ratio (SiNAD), the resolution of the analogue-todigital conversion, and the number of antenna elements for transmission and/or reception.

Baseband (BB) Interface: The baseband engine (performing digital signal processing) carries out digital up/downconversion, including filtering, FFT/IFFT for OFDM, modulation/demodulation, digital-pre-distortion (only in DL and for large BSs), signal detection (synchronization, channel estimation, equalization, compensation of RF non-idealities), and channel coding/decoding. For large BSs the digital baseband also includes the power consumed by the serial link to the backbone network. Finally, platform control and MAC operation also add a power consumer (control processor) on the platform.

The silicon technology significantly affects the power consumption of the BB interface. This technology scaling is incorporated into the power model by extrapolating on the International Technology Roadmap for Semiconductors (ITRS). The ITRS anticipates that silicon technology is replaced by a new generation every 2 years, each time doubling the active power efficiency but multiplying by 3 the leakage [12]. The increasing leakage puts a limit on the power reduction that can be achieved through technology scaling. Moreover, unlike active power, leakage does not scale with system load. Apart from the technology, the main parameters that affect the BB power consumption are related to the signal bandwidth, number of antennas, and modulation and coding schemes. The consumed power may scale linearly with most of these parameters, provided an optimized design ensures that the power is reduced at lower rates. The MIMO signal detection, however, scales more than linearly with the number of antennas.

Cooling and Power Supply may be approximated as losses that scale linearly with the power consumption of the other components.

Table I summarizes the breakdown of the state of the art BS power consumption as of 2010 at maximum load $P_{\text {out }}=P_{\max }$.
TABLE I

LTE BS POWER CONSUMPTION OF 2010 FOR DIFFERENT BS TYPES.

\begin{tabular}{|c|c|c|c|c|c|c|}
\hline & & & Macro & Micro & Pico & Femto \\
\hline$\overline{\overline{\mathbf{P A}}}$ & $\overline{P_{\max }}$ & $\overline{\overline{[\mathrm{dBm}]}}$ & $\overline{466.0}$ & $\overline{38.0}$ & $\overline{221.0}$ & $\overline{177.0}$ \\
\hline & & [W] & 39.8 & 6.3 & 0.13 & 0.1 \\
\hline & PAPR & [dB] & 8.0 & 8.0 & 12.0 & 12.0 \\
\hline & PA Efficiency & {$[\%]$} & 31.1 & 22.8 & 6.7 & 4.4 \\
\hline & $P_{\mathrm{PA}}$ & [W] & 128.2 & 27.7 & 1.9 & 1.1 \\
\hline TRX & $P_{\mathrm{TX}}$ & [W] & 6.8 & 3.4 & 0.4 & 0.2 \\
\hline & $P_{\mathrm{RX}}$ & [W] & 6.1 & 3.1 & 0.4 & 0.3 \\
\hline BB & $\begin{array}{l}\text { Radio } \\
\text { (inner Rx/Tx) }\end{array}$ & [W] & 10.8 & 9.1 & 1.2 & 1.0 \\
\hline & $\begin{array}{l}\text { Turbo code } \\
\text { (outer } \mathrm{Rx} / \mathrm{Tx} \text { ) }\end{array}$ & [W] & 8.8 & 8.1 & 1.4 & 1.2 \\
\hline & Processors & [W] & 10.0 & 10.0 & 0.4 & 0.3 \\
\hline DC-D & , loss & [\%] & 8.0 & 8.0 & 10.0 & 10.0 \\
\hline Cooli & , loss & [\%] & 12.0 & 0.0 & 0.0 & 0.0 \\
\hline Main & upply, loss & [\%] & 9.0 & 9.0 & 12.0 & 12.0 \\
\hline Total & er PA & {$[\mathrm{W}]$} & 225.0 & 72.3 & 7.3 & 5.2 \\
\hline \# Sec & & \# & 3 & 1 & 1 & 1 \\
\hline \# Ant & nas & \# & 2 & 2 & 2 & 2 \\
\hline \# Car & & \# & 1 & 1 & 1 & 1 \\
\hline Total & Radios & [W] & 1350.0 & 144.6 & 14.7 & 10.4 \\
\hline
\end{tabular}

\section{B. BS Power Consumption at Variable Load}

In a conventional $\mathrm{BS}$, the power consumption depends on the traffic load; it is mainly the PA power consumption that scales down due to reduced traffic load. This mainly happens when, e.g., the number of occupied subcarriers is reduced in idle mode operation, and/or there are subframes not carrying data. Naturally this scaling over signal load largely depends on the BS type; for macro BSs the PA accounts for $55-60 \%$ of the overall power consumption at full load, whereas for low power nodes the PA power consumption amounts to less than $30 \%$ of the total.

Fig. 3 shows BS power consumption curves for a LTE system with $10 \mathrm{MHz}$ bandwidth and $2 \times 2 \mathrm{MIMO}$ configuration. Three sectors are considered for macro BSs, whereas omnidirectional antennas are used for the smaller BS types. While the power consumption $P_{\text {in }}$ is load dependent for macro BSs, and to a lesser extent for micro BSs, there is a negligible load dependency for pico and femto BSs. The reason is that for low power BSs, the impact of the PA is diminishing. Other components hardly scale with the load in a state of the art implementation; although some more innovative designs could lead to an improved power scaling at low loads. As can be seen in Fig. 3, the relations between relative RF output power $P_{\text {out }}$ and BS power consumption $P_{\text {in }}$ are nearly linear. Hence, a linear approximation of the power model is justified:

$$
P_{\text {in }}=P_{0}+\Delta_{\mathrm{p}} P_{\text {out }}, \quad 0<P_{\text {out }} \leq P_{\text {max }}
$$

where $P_{\max }$ denotes the maximum RF output power at maximum load, $P_{0}$ is the power consumption calculated at the minimum possible output power, assumed to be $1 \%$ of $P_{\max }$, and $\Delta_{\mathrm{p}}$ is the linear factor related to load. The parameters for the different BS types are summarized in Table II.

\section{ENERgy CONSUMPTION METRICS}

To capture the energy consumption perspective in the analysis, we employ the two energy consumption indices: 

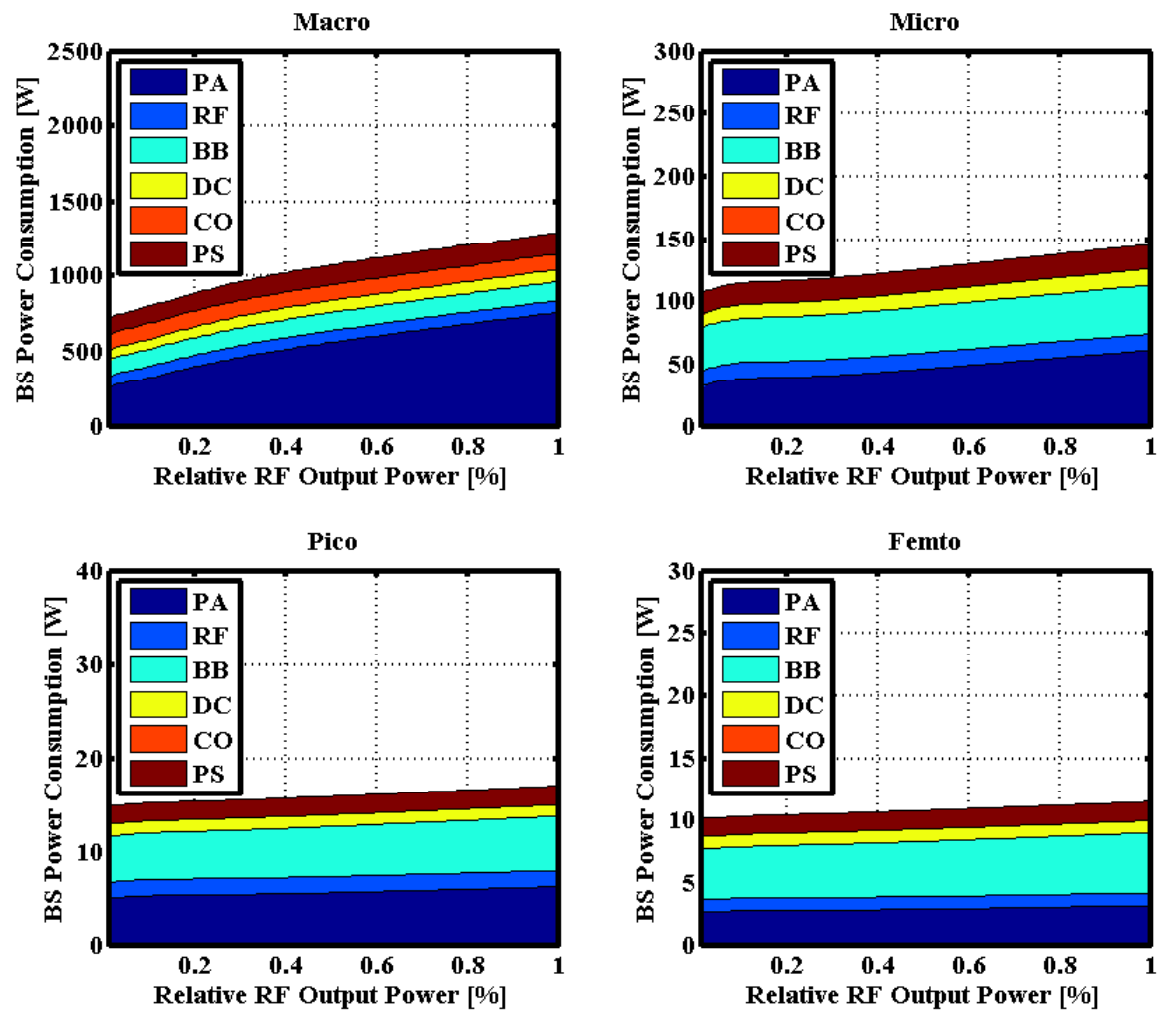

Fig. 3. Power consumption dependency on relative linear output power for various BS types for $10 \mathrm{MHz}$ system bandwidth and $2 \times 2 \mathrm{MIMO}$ configuration. Macro BSs employ 3 sectors per site. Legend: PA: Power Amplifier, RF: small signal RF transceiver, BB: Baseband processor, DC: DC-DC converters, CO: Cooling, PS: AC/DC Power Supply.

TABLE II

POWER MODEL PARAMETERS FOR DIFFERENT BS TYPES

\begin{tabular}{l|ccc} 
BS type & $P_{\max }[\mathrm{W}]$ & $P_{0}[\mathrm{~W}]$ & $\Delta_{\mathrm{p}}$ \\
\hline Macro & 40.0 & 712.0 & 14.5 \\
Micro & 6.3 & 106.0 & 6.35 \\
Pico & 0.25 & 14.9 & 8.4 \\
Femto & 0.1 & 10.1 & 15.0
\end{tabular}

- Power per area unit, measured in $\left[\mathrm{W} / \mathrm{m}^{2}\right]$;

- Energy per bit, measured in [J/bit].

The power per area unit metric is defined as the network average power usage divided by the coverage area of the network, $P / A$ and is measured by the unit $\left[\mathrm{W} / \mathrm{m}^{2}\right]$. The metric is a measure for the total energy consumption and is closely related to the $\mathrm{CO}_{2}$ emissions and the associated carbon footprint. Power per area unit is particularly relevant at low traffic loads, as in this case the network is coverage limited rather than capacity limited. This metric is also proposed in [13]; however, in ETSI this quantity is expressed as an efficiency metric, $A / P$, instead of the consumption metric adopted here.

The energy per bit metric measured in $[\mathrm{J} / \mathrm{bit}]$ is defined as the network energy consumption $E$ during the observation period $T$, divided by the total number of bits $R T$ that were correctly delivered in the network. Since the number of successfully transmitted bits is the average rate $R$ multiplied by the observation period $T$, this metric could equivalently be described as the average network power $P=E / T$ in relation to the average data rate $R$, expressed in [W/bps].

The $[\mathrm{J} / \mathrm{bit}]$ metric is commonly used in the literature, especially for theoretical studies and single link evaluations. However, in these scenarios it is used as an efficiency metric as $[\mathrm{bit} / \mathrm{J}]$.

\section{CAse Study: Energy EfFiciency of LTE}

For short-term, small-scale evaluations a macro-cellular network with regular hexagonal cell layout is implemented. 19 sites, each with 3 sectors, $10 \mathrm{MHz}$ bandwidth operating at $2.1 \mathrm{GHz}$ carrier frequency is assumed. Moreover, $2 \times 2 \mathrm{MIMO}$ transmission with adaptive rank adaption is assumed. The inter-site distance (ISD) for the dense urban and urban environments is set to $500 \mathrm{~m}$, whereas the ISD for suburban and rural areas is set to $1732 \mathrm{~m}$. Users are uniformly distributed. The simulation parameters are taken from 3GPP specifications [6].

The power per area unit $P / A$, expressed in $\left[\mathrm{kW} / \mathrm{km}^{2}\right]$, is depicted in Fig. 4 (top). The power consumption increases with the served traffic in the network. In an urban scenario, with an ISD of $500 \mathrm{~m}$ corresponding to a coverage area of $0.2165 \mathrm{~km}^{2}$ per site, the power per area unit is around $4.15 \mathrm{~kW} / \mathrm{km}^{2}$ at low loads, whereas it approaches $5.1 \mathrm{~kW} / \mathrm{km}^{2}$ at high loads. For comparison, an empty network when only control channels are transmitted, but no user data, the energy consumption equals $885 \mathrm{~W}$, which corresponds to a power per area unit of $4.1 \mathrm{~kW} / \mathrm{km}^{2}$. In the (hypothetical) extreme case, when nothing at all is transmitted (i.e., no data and no control channels) so 


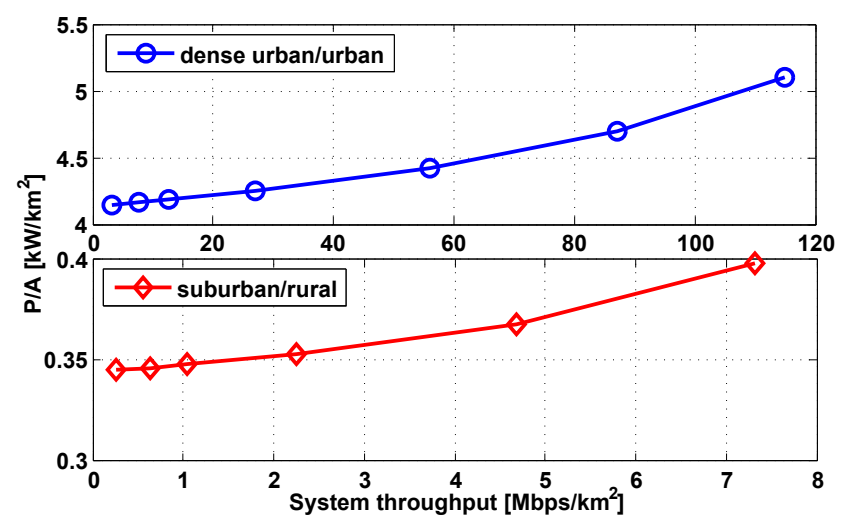

Fig. 4. Power per area unit, $P / A$, versus system throughput in dense urban/urban (top) and suburban/rural (bottom) scenarios. Notice the different scales in both $\mathrm{x}$ and $\mathrm{y}$-axis.

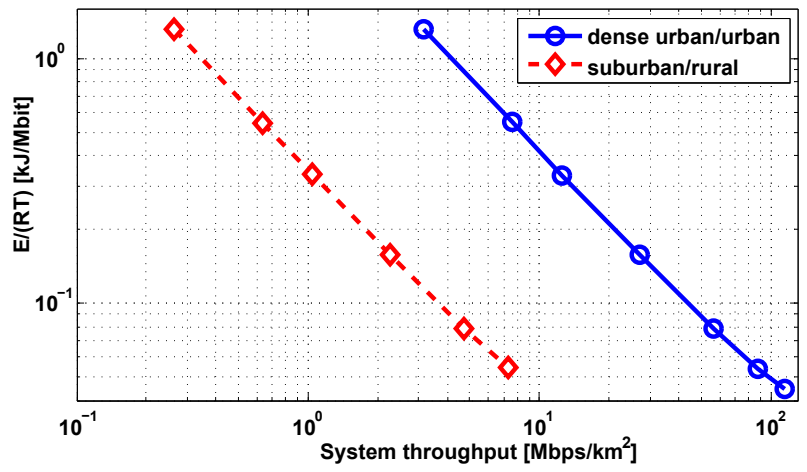

Fig. 5. Energy per bit versus system throughput in dense urban/urban (top) and suburban/rural (bottom) scenarios. Notice that both $\mathrm{x}$ and $\mathrm{y}$-axis are plotted in log-scale.

that the RF output power is $0 \mathrm{~W}$, we obtain $P / A=3.3 \mathrm{~kW} / \mathrm{km}^{2}$. The power consumption per area unit for suburban and rural areas, shown in Fig. 4 (bottom), is substantially lower, which is due to the increased ISD of $1732 \mathrm{~m}$, which corresponds to a coverage area of $2.6 \mathrm{~km}^{2}$. However, the system throughput per area unit decreases accordingly, due to the increased site coverage area.

Fig. 5 contains the energy consumption per delivered bit, $E /(R T)$ in [kJ/Mbit], over the served data rate $R$ in [Mbps]. Even though the total energy consumption increases with the traffic load, the energy consumption per bit decreases with traffic. That is, the number of delivered bits increases faster than the network energy consumption. The dominating reason for this is the fact that the power model (1) is associated with a fixed cost at $P_{\text {out }}=0 \mathrm{~W}$ RF output power and when the traffic increases, this fixed cost is shared over a larger number of bits, which results into the energy per bit decrease. Interestingly, the slope of the curves are similar for all considered scenarios, although the served data rates for given energy per bit value are significantly different.

\section{CONCLUSIONS}

In order to identify the key levers for energy savings the power consumption of mobile communication systems needs to be quantified. The most important extension to existing evaluation frameworks is a sophisticated power models that maps the radiated RF power to the supply power of a BS site. Numerical results reveal that in the current network design and operation, the power consumption is mostly independent of the traffic load. This clearly indicates that there is a vast potential for energy savings by improving the energy efficiency of BSs at low load. Among the most promising approaches are:

- Improving the power scaling of BS components, so to enhance their power efficiency at low loads.

- Reduce the amount of control signals, such as the broadcast channel and reference symbols (pilots) that facilitate channel estimation. At low loads control signals constitutes the majority of the emitted RF power.

- Energy aware transmission techniques that are optimized for low loads and that take into account the characteristics of the power amplifier and other components.

\section{ACKNOWLEDGMENTS}

This work has received funding from the European Community's $7^{\text {th }}$ Framework Programme FP7/2007-2013 under grant agreement $\mathrm{n}^{\circ} 247733$ - project EARTH.

The authors gratefully acknowledge the invaluable insights and visions received from partners of the EARTH consortium.

\section{REFERENCES}

[1] International Telecommunication Union, "Worldwide mobile cellular subscribers to reach 4 billion mark late 2008," Press release, 2008.

[2] J. Malmodin, "The importance of energy in LCA for the ICT sector", in Proc. of SETAC Europe 14th LCA Case Study Symposium, placeCityGöteborg, country-regionSweden, Dec. 2007.

[3] M. Gruber, O. Blume, D. Ferling, D. Zeller, M. A. Imran, and E. Calvanese-Strinati, "EARTH - Energy Aware Radio and Network Technologies," in Proc. IEEE Int. Symp. Personal, Indoor and Mobile Radio Communications (PIMRC), Cannes, France, 2008.

[4] J. T. Louhi and H.-O. Scheck, "Energy efficiency of cellular networks," in Proc. Int. Symp. Wireless Personal Multimedia Communications $(W P M C)$, Lapland, Finland, 2008.

[5] L. M. Correia, D. Zeller, O. Blume, D. Ferling, Y. Jading, I. Godor, G. Auer, L. Van der Perre, "Challenges and Enabling Technologies for Energy Aware Mobile Radio Networks," IEEE Communications Magazine special issue on green radio, pp. 66-72, Nov. 2010.

[6] 3GPP TR 36.814 v9.0.0, "Further advancements for E-UTRA. Physical layer aspects (Release 9)," 3GPP, Technical Specification Group Radio Access Network, Mar. 2010.

[7] 3GPP TR 25.814 v7.1.0, "Physical layer aspects for evolved Universal Terrestrial Radio Access (UTRA), (Release 7)," 3GPP, Technical Specification Group Radio Access Network, Oct. 2006.

[8] WINNER II, "D6.13.7: Test Scenarios and Calibration Cases Issues 2", Deliverable, IST-4-027756, December 2006.

[9] International Telecommunication Union, Report ITU-R M.2134, "Requirements related to technical performance for IMT-Advanced radio interface(s)," 2008, http://www.itu.int/dms_pub/itu-r/opb/rep/RREP-M.2134-2008-PDF-E.pdf

[10] K. Brueninghaus, D. Astély, T. Sälzer, S. Visuri, A. Alexiou, S. Karger, and G.-A. Seraji, "Link Performance Models for System Level Simulations of Broadband Radio Access Systems," in Proc. Int. Symp. Personal, Indoor and Mobile Radio Communications (PIMRC), Berlin, Germany, Sep. 2005, pp. 2306-2311.

[11] Jinbiao Xu, "Practical Digital Pre-Distortion Techniques for PA Linearization in 3GPP LTE", Agilent Technologies, 2010.

[12] S. Borkar, "Design Challenges of Technology Scaling," IEEE Micro, vol. 19, no. 4, pp. 23-29, July 1999.

[13] ETSI TS 102706 v1.1.1, "Environmental Engineering (EE); Energy Efficiency of Wireless Access Network Equipment," Aug. 2009. 